\title{
Communication
}

\section{Antihypertensive Effect of Long-Term Oral Administration of Jellyfish (Rhopilema esculentum) Collagen Peptides on Renovascular Hypertension}

\section{Yongliang Zhuang *, Liping Sun, Yufeng Zhang and Gaoxiang Liu}

Research Center of Food Engineering, College of Chemistry and Engineering, Kunming University of Science and Technology, Kunming, Yunnan 650600, China; E-Mails: kmlpsun@yahoo.com.cn (L.S.); zhangyufeng062@163.com (Y.Z.); 1gx1110@126.com (G.L.)

* Author to whom correspondence should be addressed; E-Mail: kmylzhuang@yahoo.com.cn; Tel./Fax: +86-871-3801122.

Received: 25 December 2011; in revised form: 31 January 2012 / Accepted: 6 February 2012 / Published: 15 February 2012

\begin{abstract}
Antihypertensive effect of long-term oral administration of jellyfish (Rhopilema esculentum) collagen peptides (JCP) on renovascular hypertension rats (RVHs) was evaluated. The systolic blood pressure and diastolic blood pressure of the RVHs were significantly reduced with administration of JCP $(p<0.05)$, compared with model control group. However, the arterial blood pressure of normal rats showed no significant changes during long-term oral treatment with high dose JCP $(p>0.05)$. Furthermore, effect of JCP on angiotensin II (Ang II) concentration of plasma had no significance $(p>0.05)$, but JCP significantly inhibited the Ang II concentration in RVHs' kidney $(p<0.05)$. The kidney should be the target site of JCP.
\end{abstract}

Keywords: jellyfish; collagen peptide; blood pressure; angiotensin II; kidney

\section{Introduction}

Hypertension is one of the most common lifestyle-related diseases and has become one of the most significant problems in recent years [1]. Angiotensin-I-converting enzyme (ACE) plays an important physiological role in regulating blood pressure. It raises blood pressure by converting the inactive decapeptide angiotensin-I (Ang I) to the potent vasoconstrictor octapeptide angiotensin-II (Ang II), as 
well as inactivating the vasodilating nonapeptide [2]. Therefore, it is feasible to suppress blood pressure elevation by inhibiting the catalytic action of ACE.

Synthetical ACE inhibitors, such as captopril, enalapril, and lisinopril, are effective for decreasing blood pressure. However, some undesirable side effects have been reported, including coughing, dizziness, headache, abnormal taste, and kidney and liver problems [3]. Therefore, safer alternatives are desirable. Recently, many peptides that exert ACE-inhibitory activity have been isolated from food-derived marine protein hydrolysates, such as cod [4], sea cucumber collagen [5], shrimp [6], salmon [7], and squid skin [8].

Antihypertensive peptides must be resistant to in vivo proteolysis once they are within the body, in order to reach the desired target tissue or organ and exert an antihypertensive effect [9]. Therefore, it is necessary to confirm the antihypertensive effect of orally administrated peptides on renovascular hypertension rats (RVHs) or spontaneously hypertensive rats (SHR). The antihypertensive effects in vivo of peptides prepared from various sources have been reported in previous works [5,10,11], but most of them did short-term experiments with rats within $24 \mathrm{~h}$. In the present study, a long-term antihypertensive experiment was performed.

Previously, we isolated and purified peptides from jellyfish collagen (JCP). The $\mathrm{IC}_{50}$ of ACE-inhibitory activity of JCP was $43 \mu \mathrm{g} / \mathrm{mL}$ in vitro [1]. In this study, the antihypertensive of JCP was determined by RVHs in vivo. The effect of JCP on the blood pressures and heart rates was investigated. Furthermore, the concentrations of Ang II in plasma and kidney were quantitated.

\section{Results and Discussion}

\subsection{Blood Pressure and Heart Rate}

Antihypertensive effect of JCP was evaluated by measuring changes in systolic blood pressure (SBP) and diastolic blood pressure (DBP) of RVHs. Figure 1 showed the SBP and DBP obtained after 1 month of oral administration of the different products, respectively. Compared with MC group, the average SBP reductions in all groups were, in descending order: Captopril group (65.2 $\mathrm{mmHg}), \mathrm{JCP}-2$ group $(55.5 \mathrm{mmHg})$ and $\mathrm{JCP}-1$ group $(32.0 \mathrm{mmHg})$. The antihypertensive effect improved with a JCP dosage increase. The average DBP of JCP-1 group $(120.2 \mathrm{mmHg})$ and JCP-2 group $(102.4 \mathrm{mmHg})$ decreased by 34.8 and $52.6 \mathrm{mmHg}$ compared with $\mathrm{MC}$ group, respectively. DBP showed no significant difference between JCP-2 group and captopril group $(p>0.05)$. Interestingly, the SBP and DBP of JCP-C group showed no significant changes during long-term oral treatment with high dose JCP $(p>0.05)$. These results indicated that JCP had good long-term antihypertensive effects on RVHs, but had no effects for the blood pressures of normal rats.

As shown in Figure 2, the heart rates of the MC group were significant higher than the captopril group and NC group $(p<0.05)$. The heart rate of JCP-C group was not different from NC group $(p>0.05)$, and the heart rats of JCP-2 group had no significance with captopril group and NC group $(p>0.05)$. 
Figure 1. Effect of jellyfish (Rhopilema esculentum) collagen peptides (JCP) on systolic blood pressure (SBP) (a) and diastolic blood pressure (DBP) (b) of renovascular hypertension rats (RVHs) after oral administration for 30 days. Values are mean \pm SD $(n=6)$. Different letter indicated significant differences $(p<0.05)$. MC group: laparotomy and isolation of the left renal artery with clip placement $(2 \mathrm{~K} 1 \mathrm{C})$ with oral administration of $0.9 \%$ saline solution; JCP-1 group: $2 \mathrm{~K} 1 \mathrm{C}$ with oral administration of JCP $(25 \mathrm{mg} / \mathrm{kg} \mathrm{bw})$; JCP-2 group: 2K1C with oral administration of JCP (100 mg/kg bw); Captopril group: $2 \mathrm{~K} 1 \mathrm{C}$ with oral administration of captopril (5 mg/ $\mathrm{kg} \mathrm{bw}$ ); $\mathrm{NC}$ group: sham surgery (laparotomy and isolation of the left renal artery without clip placement) with oral administration of $0.9 \%$ saline solution; JCP-C group: sham surgery with oral administration of JCP (100 mg/kg bw).

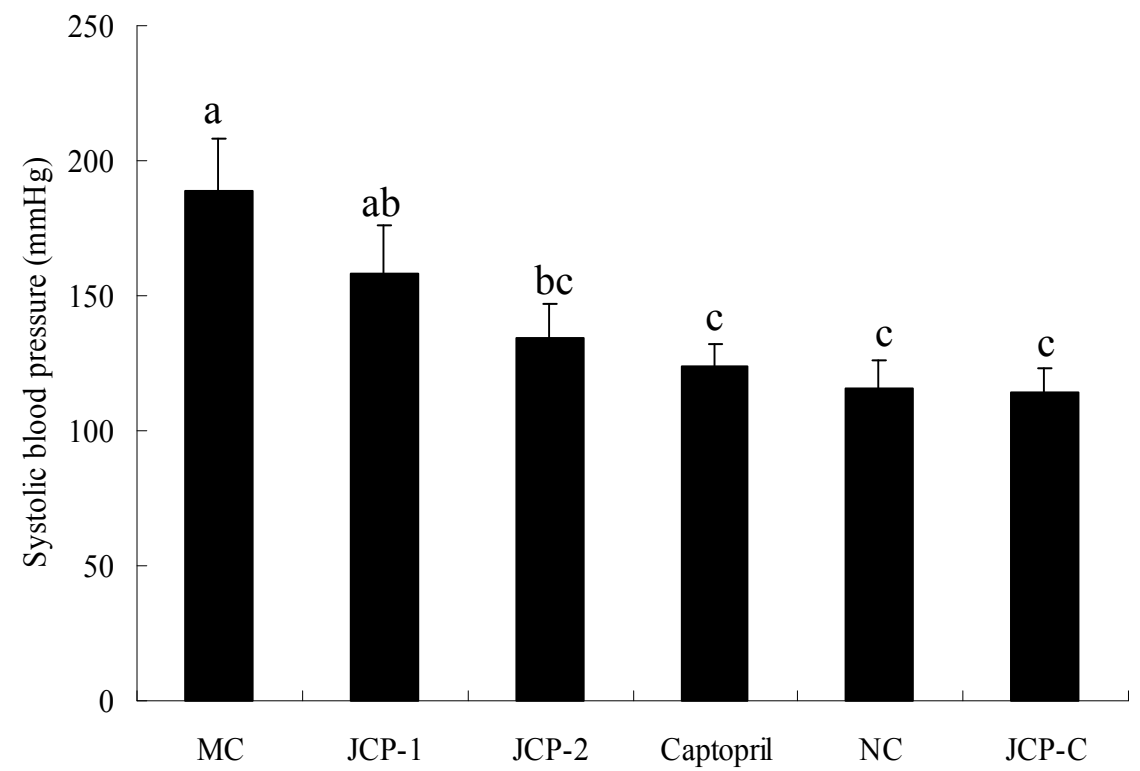

(a)

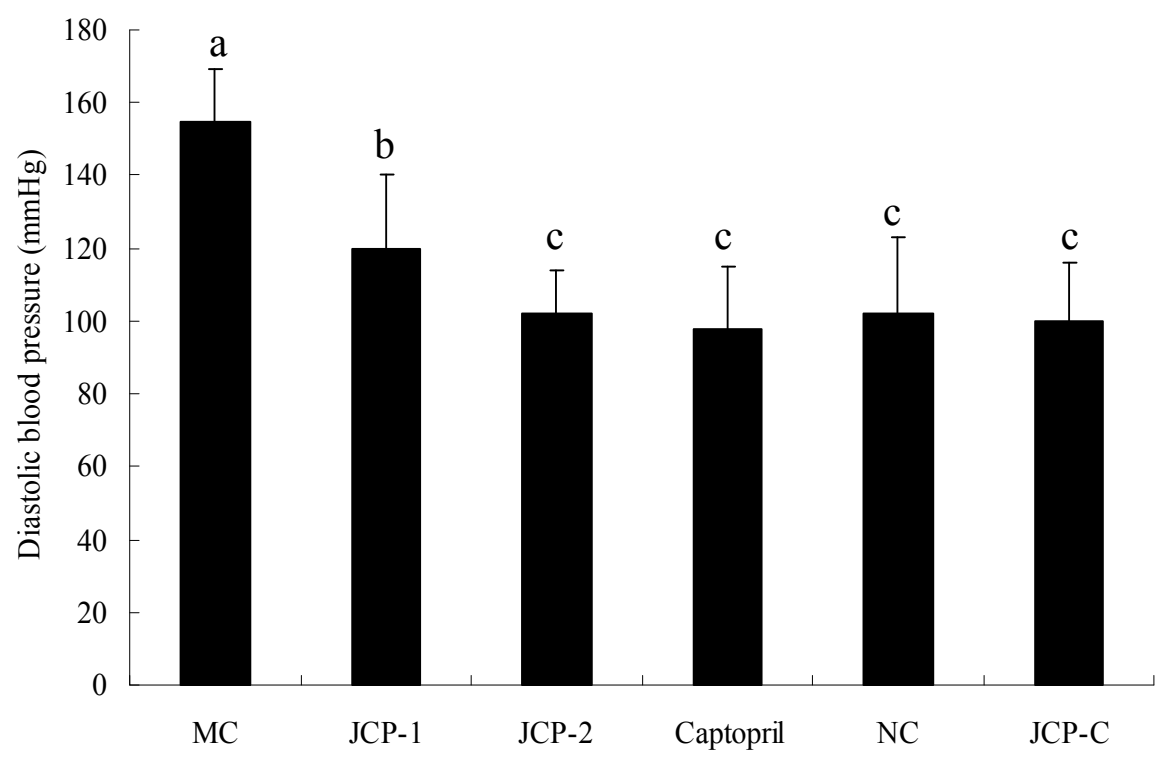

(b) 
Figure 2. Effect of JCP on heart rate of RVHs after oral administration for 30 days. Values are mean $\pm \mathrm{SD}(n=6)$. Different letter indicated significant differences $(p<0.05)$. MC group: laparotomy and isolation of the left renal artery with clip placement $(2 \mathrm{~K} 1 \mathrm{C})$ with oral administration of $0.9 \%$ saline solution; JCP-1 group: $2 \mathrm{~K} 1 \mathrm{C}$ with oral administration of JCP (25 mg/kg bw); JCP-2 group: 2K1C with oral administration of JCP (100 mg/kg bw); Captopril group: 2K1C with oral administration of captopril ( $5 \mathrm{mg} / \mathrm{kg} \mathrm{bw})$; $\mathrm{NC}$ group: sham surgery (laparotomy and isolation of the left renal artery without clip placement) with oral administration of $0.9 \%$ saline solution; JCP-C group: sham surgery with oral administration of JCP $(100 \mathrm{mg} / \mathrm{kg} \mathrm{bw})$.

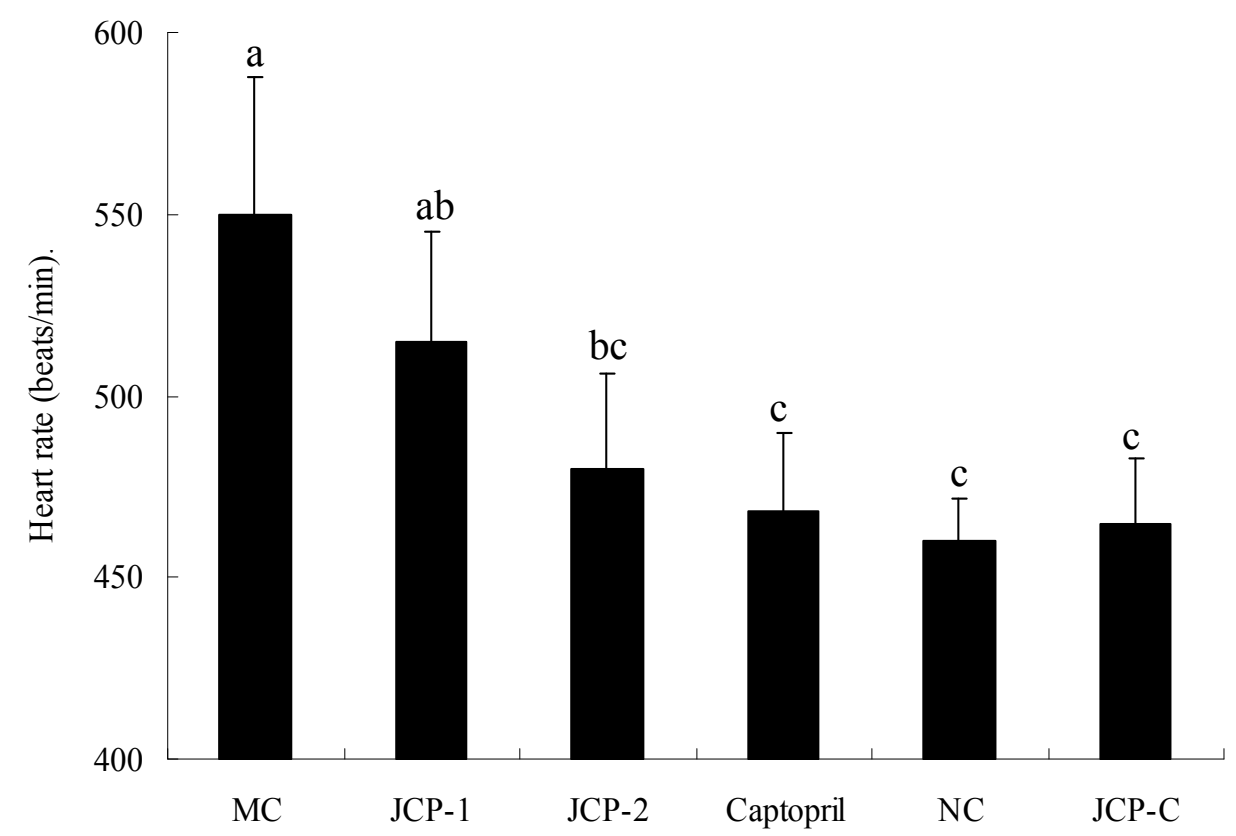

Human tissues are exposed to bioactive peptides via the systemic circulation. However, before bioactive peptides reach tissues, peptides are substantially hydrolyzed during small intestinal passage and absorption [12]. The digestive system in vivo is capable of handling a wide range of protein sources and the cascade of gastrointestinal proteolytic and peptidolytic enzymes very efficiently degrades proteins from their quaternary structure into single amino acids. Some peptides failed to show antihypertensive activity after oral administration to spontaneously hypertensive rats, in spite of their in vitro activity [13]. Because peptides are prone to extensive hydrolysis in the gastrointestinal tract by stomach, small intestinal, and brush border peptidases, so bioactive peptides must be absorbed from the intestine intact and be resistant to degradation by plasma peptidases to reach the target sites [14], so antihypertensive peptides have limited application if they have no oral activity [15]. Phe-Lys-Gly-Arg-Tyr-Tyr-Pro isolated from the thermolysin digest of chicken muscle showed an in vitro $\mathrm{IC}_{50}$ value of $0.55 \mathrm{mM}$, but no antihypertensive activity could be observed after oral administration to SHR [16]. Vercruysse et al. studied enzymatic hydrolysates of insect protein had high ACE inhibitory activity [17]. Ala-Val-Phe and Val-Phe were the key peptides in the hydrolysates. The ACE inhibitory activity of Val-Phe was higher than that of Ala-Val-Phe, and in organ bath experiments using rat aorta, Val-Phe showed ACE inhibitory, while Ala-Val-Phe did not. However, single oral administration to spontaneously hypertensive rats led to a significant decrease in blood 
pressure for both peptides, because Val-Phe was released by in vivo peptidases from Ala-Val-Phe [18]. Our previous study showed JCP had potent ACE inhibitory activity in vitro with an $\mathrm{IC}_{50}$ value of $43 \mu \mathrm{g} / \mathrm{mL}$ [1]. In this study, antihypertensive activity of JCP was evaluated in vivo. After the oral administration of JCP, the SBP and DBP of RVHs significantly decreased $(p<0.05)$, indicating that JCP had an intense effect on the reduction of blood pressure in vivo. Therefore, some peptides of JCP may have resistance to gastrointestinal enzymes and could be absorbed in their intact active form to lower the blood pressure.

\subsection{Angiotensin II Concentration}

The Ang II concentrations of the plasma and kidney were investigated. Figure 3 depicted changes in the plasma and kidney Ang II levels after the JCP dosing. The concentration of Ang II in plasma had no significance during the period $(p>0.05)$. This suggested that JCP was not directly associated with an inhibition of plasma ACE. Compared with NC group, JCP-1 group and JCP-2 group significantly decreased Ang II concentration in kidney during long-term oral treatment ( $p<0.05$ ), and JCP-2 group had no significance with captopril group and NC group $(p>0.05)$. Interestingly, during long-term oral treatment with high dose JCP $(100 \mathrm{mg} / \mathrm{kg})$, the Ang II concentrations of the plasma and kidney of JCP-C group were not different from NC group $(p>0.05)$.

Figure 3. Effect of JCP on the Ang II concentrations of plasma (a) and kidney (b) of RVHs after oral administration for 30 days. Values are mean $\pm \operatorname{SD}(n=6)$. Different letter indicated significant differences $(p<0.05)$. MC group: laparotomy and isolation of the left renal artery with clip placement $(2 \mathrm{~K} 1 \mathrm{C})$ with oral administration of $0.9 \%$ saline solution; JCP-1 group: $2 \mathrm{~K} 1 \mathrm{C}$ with oral administration of JCP (25 mg/kg bw); JCP-2 group: $2 \mathrm{~K} 1 \mathrm{C}$ with oral administration of JCP (100 mg/kg bw); Captopril group: $2 \mathrm{~K} 1 \mathrm{C}$ with oral administration of captopril ( $5 \mathrm{mg} / \mathrm{kg} \mathrm{bw}$ ); $\mathrm{NC}$ group: sham surgery (laparotomy and isolation of the left renal artery without clip placement) with oral administration of $0.9 \%$ saline solution; JCP-C group: sham surgery with oral administration of JCP (100 mg/kg bw).

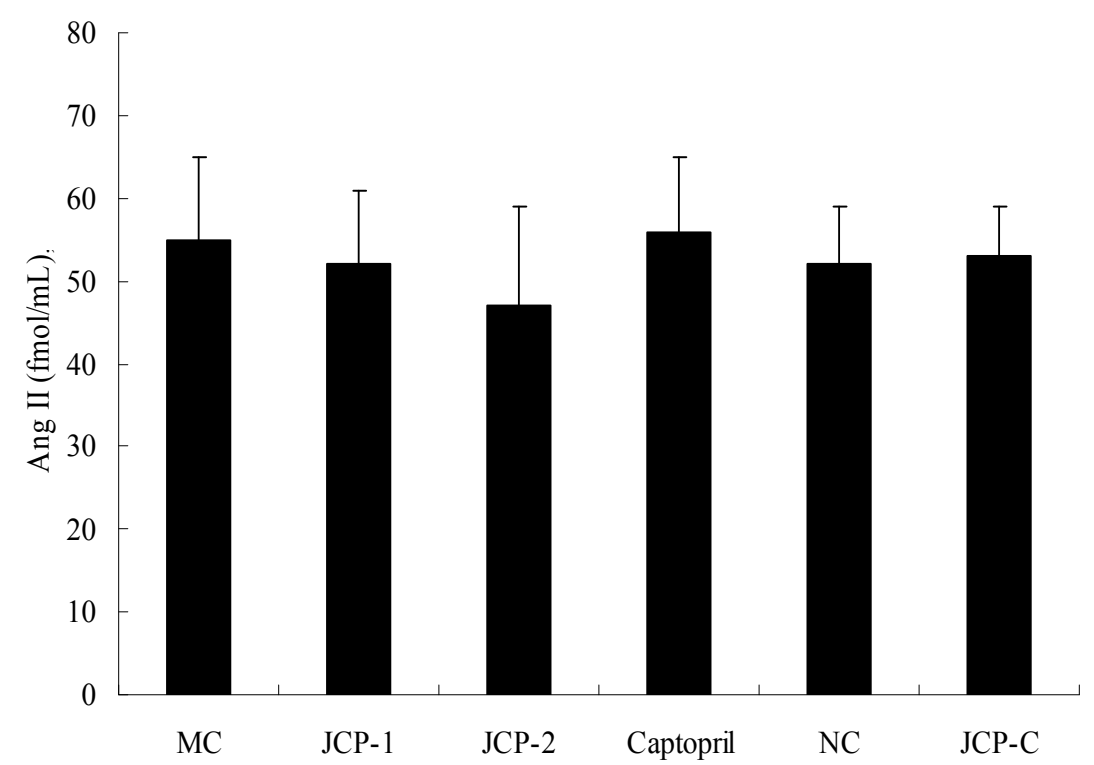

(a) 
Figure 3. Cont.

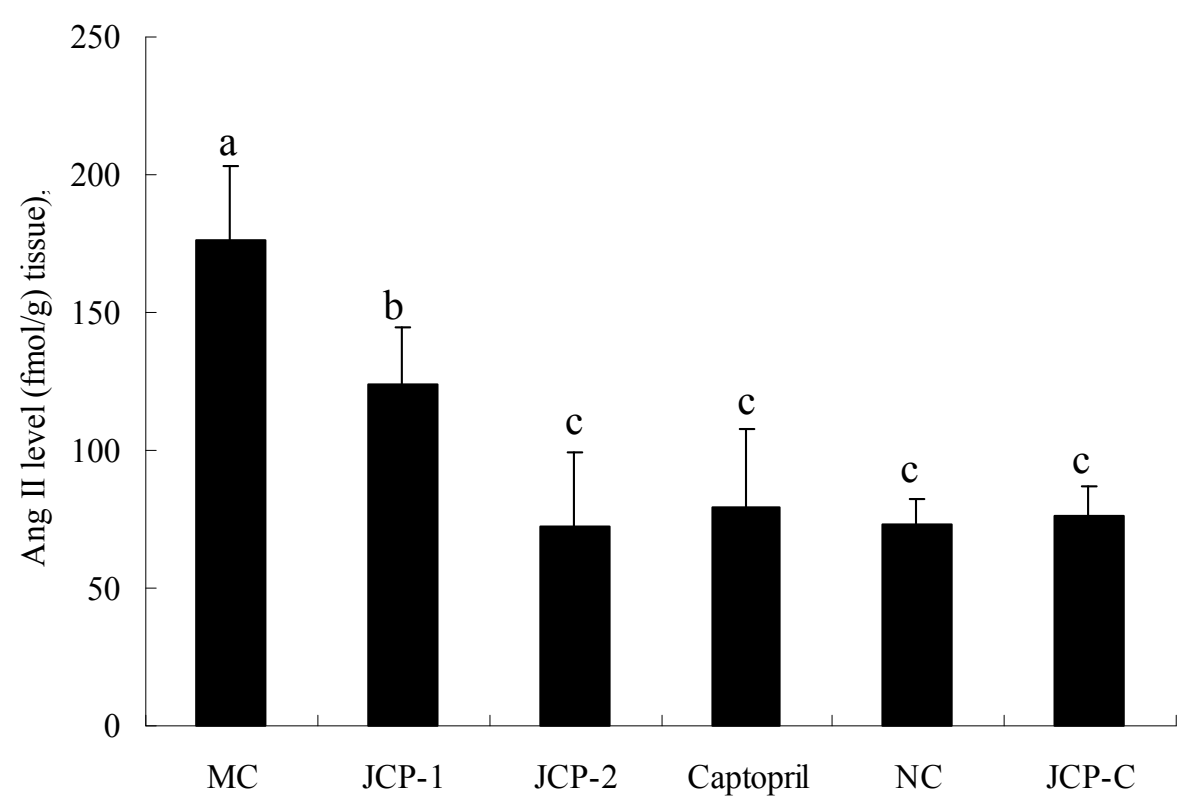

(b)

Classically, the rennin-angiotensin system plays an important role in regulating arterial pressure [19]. ACE is a nonspecific dipeptidyl carboxypeptidase associated with it. This enzyme increases blood pressure by converting the Ang I into the Ang II [20]. Ang II has many important actions, including increasing arterial pressure, increasing sodium and fluid retention, enhancing sympathetic adrenergic function and causing cardiac and vascular remodeling [21]. Inhibition of ACE is believed to lower blood pressure, since it reduces the production of Ang II. Experimental Renovascular Hypertension is characterized by significant up-regulation of intrarenal Ang II levels, which is believed to play an important pathogenetic role in the development and maintenance of hypertension in RVH. Researchers thought that the prolonged antihypertensive action of ACE inhibitors might be related to persistent inhibition of ACE activity and Ang II concentrations of tissues, such as vascular wall and kidneys [22,23]. The antihypertensive activities of captopril are known to be related to the inhibition of ACE activities and Ang II concentration in such organs as the lung, kidney, aorta, and brain [24]. The present study investigated the role of Ang II production in the plasma and kidney. Our results showed the concentration of Ang II in plasma had no significance during long-term oral administration JCP ( $p>0.05$ ), but the concentration of Ang II in kidney significantly decreased in JCP groups $(p<0.05)$. So, the kidney should be the target site of JCP. Miguel et al. [25] indicated that ACE inhibitors with different molecular structures had different metabolism pathways and tissue distribution. These characteristics determined an ACE inhibitory effect in tissue and thus exhibited different antihypertensive effects. Lin et al. [8] reported the administration of Captopril produced a significant decrease in plasma concentrations of Ang II $(p<0.05)$, but squid skin gelatin hydrolysates had no statistically significance $(p>0.05)$. Matsui et al. [26] had demonstrated that Val-Tyr greatly affected tissue Ang II in the lung, heart, kidney, aorta and mesenteric artery, whereas no suppression was observed in the plasma. 


\section{Experimental Section}

\subsection{Materials}

Jellyfish collagen peptides (JCP) were prepared by the previous study. Captopril was purchased from Sigma Chemical Co. (St. Louis, MO). All other reagents used in this study were analytical grade.

\subsection{Animal Treatment}

The Wistar strain male rats (SPF, $150 \pm 20 \mathrm{~g})$ were fed ad libitum and housed under conventional conditions at a controlled temperature $\left(23 \pm 2{ }^{\circ} \mathrm{C}\right)$, humidity $(55 \pm 10 \%)$, and light $(12 \mathrm{~h}$ light/12 h darkness). All animal treatments were strictly in accordance with international ethical guidelines and the National Institutes of Health Guide on the Care and Use of Laboratory Animals. A two-kidney, one-clip (2K1C) rat model was used to induce RVH. A midline laparotomy was used for placement of a partially occlusive, a silver clip (0.2 mm internal diameter) on the left renal artery of 2K1C rats [27].

The rats were grouped as model control (MC), negative control (NC), positive control (captopril), JCP-1 (25 mg/kg bw), JCP-2 (100 mg/kg bw) and JCP-C (100 mg/kg bw) ( $n=6$ in each group). Sham surgery (laparotomy and isolation of the left renal artery without clip placement) was used as a negative control and JCP-C group underwent the sham surgery with oral administration of JCP $(100 \mathrm{mg} / \mathrm{kg} \mathrm{bw})$. NC group and MC group were administrated with $0.9 \%$ saline solution $(5 \mathrm{~mL} / \mathrm{kg} \mathrm{bw})$. Captopril ( $5 \mathrm{mg} / \mathrm{kg} \mathrm{bw}$ ), a known ACE inhibitor, served as a positive control group. JCP-1 group $(25 \mathrm{mg} / \mathrm{kg} \mathrm{bw}), \mathrm{JCP}-2$ group $(100 \mathrm{mg} / \mathrm{kg} \mathrm{bw})$ and JCP-C group (100 mg/kg bw) were daily given the JCP dissolved in the same volume of saline solution via gastric intubation for 30 days.

\subsection{Measurement of Blood Pressure and Heart Rate}

Thirty days after oral sample administrations, the rats were anesthetised with sodium pentobarbital $(40 \mathrm{mg} / \mathrm{kg} \mathrm{bw})$. A catheter was inserted into the left carotid artery to measure mean arterial pressure and heart rate with a pressure transducer coupled to computer recorder (GY-6088, Kaifeng-Huanan, China).

\subsection{Determination of Angiotensin-II (Ang II) Concentration}

The plasma and kidney were collected to measure Ang II concentration. The concentrations of Ang II were determined by Ang II radioimmunoassay kits (Beijing North Institute of Biological Technology, China) [8].

\subsection{Statistical Analysis}

Results were expressed as the mean \pm SD. Data were analyzed by one-way analysis of variance (ANOVA) using SPSS (version 11.0, Chicago, IL, USA) and the differences between the means assessed using Duncan's multiple range test. A $p$ value of $<0.05$ was taken as the level of statistical significance. 


\section{Conclusions}

Antihypertensive effect of long-term oral administration of jellyfish collagen peptides (JCP) on renovascular hypertension rats (RVHs) was evaluated in vivo in this study. The results showed JCP was a safe selection to decrease blood pressure. After oral administration of JCP, the systolic blood pressure and diastolic blood pressure of RVHs significantly decreased $(p<0.05)$, indicating that JCP had an intense effect on the reduction of blood pressure in vivo. Furthermore, JCP showed antihypertensive effects through inhibiting the Ang II concentrations in the kidney, and the kidney should be the target site of JCP.

\section{Acknowledgements}

We gratefully thank the National Natural Science Foundation of China (Grant No. 31101392) for the financial support on this research.

\section{References}

1. Zhuang, Y.; Sun, L.; Li, B. Production of the angiotensin-I-converting enzyme (ACE)-inhibitory peptide from hydrolysates of jellyfish (Rhopilema esculentum) collagen. Food Bioprocess Technol. 2010, doi:10.1007/s11947-010-0439-9.

2. Richard, J.F.; Brain, A.M.; Daniel, J.W. The emerging role of dairy protein and bioactive peptides in nutrition and health. J. Nutr. 2004, 134, 980-988.

3. Huang, W.H.; Sun, J.; He, H.; Dong, H.W.; Li, J.T. Antihypertensive effect of corn peptides, produced by a continuous production in enzymatic membrance reactor, in spontaneously hypertensive rats. Food Chem. 2011, 128, 968-973.

4. Kim, S.K.; Choi, Y.R.; Park, P.J.; Choi, J.H.; Moon, S.H. Screening of biofunctional peptides from cod processing wastes. J. Korean Soc. Agric. Chem. Biotechnol. 2000, 43, 225-227.

5. Zhao, Y.H.; Li, B.F.; Dong, S.Y.; Liu, Z.Y.; Zhao, X.; Wang, J.F.; Zeng, M.Y. A novel ACE inhibitory peptide isolated from Acaudina molpadioidea hydrolysate. Peptides 2009, 30, 1028-1033.

6. Zhang, C.H.; Cao, W.H.; Hong, P.Z.; Ji, H.W.; Qin, X.M.; He, J.F. Angiotensin converting enzyme inhibitory activity of Acetes chinensis peptic hydrolysate and its antihypertensive effect in spontaneously hypertensive rats. Int. J. Food Sci. Technol. 2009, 44, 2042-2048.

7. Ono, S.; Hosokawa, M.; Miyashita, K.; Takahashi, K. Inhibition properties of dipeptides from salmon muscle hydrolysate on angiotensin I-converting enzyme. Int. J. Food Sci. Technol. 2006, 41, 383-386.

8. Lin, L.; Shun, L.; Li, B.F. Angiotensin-I-converting enzyme (ACE)-inhibitory and antihypertensive properties of squid skin gelatin hydrolysates. Food Chem. 2011, doi:10.1016/j.foodchem.2011.08.064.

9. Wu, J.; Aluko, R.E.; Nakai, S. Structural requirements of angiotensin I converting enzyme inhibitory peptides: Quantitative structure-activity relationship study of di- and tripeptides. J. Agric. Food Chem. 2006, 54, 732-738. 
10. Herregods, G.; van Camp, J.; Morel, N.; Ghesquière, B.; Gevaert, K.; Vercruysse, L.; Dierckx, S.; Quanten, E.; Smagghe, G. Angiotensin I-converting enzyme inhibitory activity of gelatin hydrolysates and identification of bioactive peptides. J. Agric. Food Chem. 2011, 59, 552-558.

11. Lee, S.H.; Qian, Z.J.; Kim, S.K. A novel angiotensin I converting enzyme inhibitory peptide from tuna frame protein hydrolysate and its antihypertensive effect in spontaneously hypertensive rats. Food Chem. 2010, 118, 96-102.

12. Foltz, M.; van der Pijl, P.C.; Duchateau, G.S.M.J.E. Current in vitro testing of bioactive peptides is not valuable. J. Nutr. 2010, 140, 117-118.

13. Maeno, M.; Yamamoto, N.; Takano, T. Identification of an antihypertensive peptide from casein hydrolysate produced by a proteinase from Lactobacillus helveticus CP790. J. Dairy Sci. 1996, 79, 1316-1321.

14. Ondetti, M.A.; Cushman, D.W. Enzymes of the rennin angiotensin system and their inhibitors. Ann. Rev. Biochem. 1982, 51, 283-308.

15. Brown, N.J.; Vaughan, D.E. Angiotensin-converting enzyme inhibitors. Circulation 1998, 97, 1411-1420.

16. Fujita, H.; Yokoyama, K.; Yoshikawa, M. Classification and antihypertensive activity of angiotensin I-converting enzyme inhibitory peptides derived from food proteins. J. Food Sci. 2000, 65, 564-569.

17. Vercruysse, L.; Smagghe, G.; Herregods, G.; van Camp, J. ACE inhibitory activity in enzymatic hydrolysates of insect protein. J. Agric Food. Chem. 2005, 53, 5207-5211.

18. Vercruysse, L.; Van Camp, J.; Morel, N.; Rougé, P.; Herregods, G.; Smagghe, G. Ala-Val-Phe and Val-Phe: ACE inhibitory peptides derived from insect protein with antihypertensive activity in spontaneously hypertensive rats. Peptides 2010, 31, 482-488.

19. Dostal, D.E.; Baker, K.M. The cardiac rennin-angiotensin system conceptual, or a regulator of cardiac function? Circ. Res. 1999, 85, 643-650.

20. Vermeirssen, V.; van Camp, J.; Verstraete, W. Optimisation and validation of an angiotensin-converting enzyme inhibition assay for the screening of bioactive peptides. J. Biochem. Biophys. Meth. 2002, 51, 75-87.

21. Fang, H.; Luo, M.; Sheng, Y.; Li, Z.X.; Wu, Y.Q.; Liu, C. The antihypertensive effect of peptides: A novel alternative to drugs? Peptides 2008, 20, 1062-1071.

22. Masuda, O.; Nakamura, Y.; Takano, T. Antihypertensive peptides are present in aorta after oral administration of sour milk containing these peptides to spontaneously hypertensive rats. Nutrition 1996, 126, 3063-3068.

23. Van der Pij1, P.C.; Kies, A.K.; Ten Have, G.A.; Duchateau, G.S.; Deutz, N.E. Pharmacokinetics of proline-rich tripeptides in the pig. Peptides 2008, 29, 2196-2202.

24. Unger, T.H.; Ganten, D.; Lang, R.E.; Schölkens, B.A. Persistent tissue converting enzyme inhibition following chronic treatment with Hoe498 and MK421 in spontaneously hypertensive rats. J. Cardiovasc. Pharmacol. 1985, 7, 36-41.

25. Miguel, M.; Contreras, M.M.; Recio, I.; Aleixandre, A. ACE-inhibitory and antihypertensive properties of a bovine casein hydrolysate. Food Chem. 2009, 112, 211-214. 
26. Matsui, T.; Imamura, M.; Oka, H.; Osajima, K.; Kimoto, K.I.; Kawasaki, T.; Matsumoto, K. Tissue distribution of antihypertensive dipeptide, Val-Tyr, after its single oral administration to spontaneously hypertensive rats. J. Peptide Sci. 2004, 10, 535-545.

27. Sadjadi, J.; Kramer, G.L.; Yu, C.H.; Welborn, M.B.; Chappell, M.C.; Modrall, J.G. Angiotensin converting enzyme-independent angitotensin II production by chymase is up-regulated in the ischemic kidney in renovascular hypertension. J. Surg. Res. 2005, 127, 65-69.

Samples Availability: Available from the authors.

(C) 2012 by the authors; licensee MDPI, Basel, Switzerland. This article is an open access article distributed under the terms and conditions of the Creative Commons Attribution license (http://creativecommons.org/licenses/by/3.0/). 